\title{
Friedel Oscillations and Charge Density Waves in Chains and Ladders
}

\author{
Steven R. White \\ Department of Physics and Astronomy, University of California, Irvine, CA 92697 \\ Ian Affleck \\ Physics Department, Boston University, 590 Commonwealth Ave., Boston, MA02215 \\ Douglas J. Scalapinof \\ Department of Physics, University of California, Santa Barbara, CA 93106-9530 USA
}

(Dated: June 8, 2018)

\begin{abstract}
The density matrix renormalization group method for ladders works much more efficiently with open boundary conditions. One consequence of these boundary conditions is groundstate charge density oscillations that often appear to be nearly constant in magnitude or to decay only slightly away from the boundaries. We analyse these using bosonization techniques, relating their detailed form to the correlation exponent and distinguishing boundary induced generalized Friedel oscillations from true charge density waves. We also discuss a different approach to extracting the correlation exponent from the finite size spectrum which uses exclusively open boundary conditions and can therefore take advantage of data for much larger system sizes. A general discussion of the Friedel oscillation wave-vectors is given, and a convenient Fourier transform technique is used to determine it. DMRG results are analysed on Hubbard and $t-J$ chains and 2 leg $t-J$ ladders. We present evidence for the existence of a long-ranged charge density wave state in the $t-J$ ladder at a filling of $n=0.75$ and near $J / t \approx 0.25$.
\end{abstract}

\section{INTRODUCTION}

Bosonization analyses] numerical work using the DMRG5 and other methods have given a clear understanding of the behavior of the 2-leg Hubbard and $t-J$ ladder models. The Hubbard Hamiltonian is written:

$$
\begin{aligned}
& H=-t \sum_{i, \lambda, \alpha}\left(c_{i+1 \lambda \alpha}^{\dagger} c_{i \lambda \alpha}+\text { h.c. }\right) \\
& -t \sum_{i, \alpha}\left(c_{i 2 \alpha}^{\dagger} c_{i 1 \alpha}+\text { h.c. }\right)+U \sum_{i, \lambda} n_{i, \lambda, \uparrow} n_{i, \lambda, \downarrow}
\end{aligned}
$$

Here $c_{i \lambda \alpha}$ destroys an electron on rung $i$ and leg $\lambda=1,2$ with spin $\alpha=\uparrow, \downarrow$. $n_{i, \lambda, \sigma}$ is the electron number operator. The $t-J$ Hamiltonian is:

$$
\begin{aligned}
H= & -t \sum_{i, \lambda, \alpha}\left(c_{i+1 \lambda \alpha}^{\dagger} c_{i \lambda \alpha}+\text { h.c. }\right) \\
& -t \sum_{i, \alpha}\left(c_{i 2 \alpha}^{\dagger} c_{i 1 \alpha}+\text { h.c. }\right) \\
& +J \sum_{i, \lambda}\left(\vec{S}_{i \lambda} \cdot \vec{S}_{i+1 \lambda}-\frac{n_{i \lambda} n_{i+1 \lambda}}{4}\right) \\
& +J \sum_{i}\left(\vec{S}_{i 1} \cdot \vec{S}_{i 2}-\frac{n_{i 1} n_{i 2}}{4}\right)
\end{aligned}
$$

where $\vec{S}_{i \lambda}=c_{i \lambda}^{\dagger} \vec{\sigma} / 2 c_{i \lambda}$ and the Hilbert space now excludes all states with doubly-occupied sites. We will generally set $t=1$ in what follows. Both models are expected to be in a "C1S0" phase, over a wide range of parameters, in which the low energy degrees of freedom consist of a single free massless charge boson, whose excitations carry even multiples of the electron charge. This is often characterized as a "d-wave superconductor" based on the nature of the power law decay of pair correlations which have a positive sign for singlet rung-rung or leg-leg correlations and a negative sign for rung-leg correlations. Both bosonization and DMRG results exist on multi-leg ladders with analytical and numerical uncertainty which increase with the number of legs.

Most of the DMRG data is obtained with open boundary conditions along the legs of the ladder. Unlike with periodic boundary conditions, this generally leads to charge density oscillations in the groundstate. If these oscillations persist at the center of the chain for arbitrarily long chain length then they correspond to a charge density wave (CDW). More commonly, they decay away from the boundaries with a power law, in the limit of an infinite chain. In this case, we may think of them as generalized Friedel oscillations where the chain ends themselves act as impurities which induce gradually decaying density oscillations. Unlike in a Fermi liquid, these generalized Friedel oscillations in a Luttinger liquid decay with an exponent which depends on the interaction strength, and which is simply related to the density correlation exponent 8.6 Furthermore, the wave-vector of the oscillations itself can be changed by the interactions. These density oscillations, for the largest systems studigd, up to eight legs, have been identified with "stripes" 10 An understanding of the occurance of stripes in these models, whether or not they require long range Coulomb interactions to exist and their connection with superconducitivity are important open problems. Since any ladder system is ultimately one-dimensional if the number of legs is held fixed and the length taken to $\infty$, the Friedel oscillations /CDW analysis may provide the appropriate 
description of stripe behavior, at least in this limit and in cases where the stripe wave-vector is parallel to the chains.

In Sec. II we briefly review the bosonization picture of the 2-leg $t-J$ ladder. We also present a technique for extracting the correlation exponent purely from data with open boundary conditions which has not, to our knowledge, been used previously on ladders, although it has been used on chains. This method is used to obtain values for the charge velocity and exponent. In Sec. III we review the bosonization treatment of commensurate charge density waves and the related behavior of the correlation exponent, and analyse data on the 2 leg tJ model at electron density $n=3 / 4$, showing that a CDW may occur for weak enough $J$. In Sec. IV we review Friedel oscillations in Luttinger liquids and apply this analysis to data on the single chain Hubbard and $t-J$ model and the 2 leg $t-J$ model.

\section{THE 2-LEG LADDER}

A convenient starting point for bosonization is the weak coupling Hubbard model version of the 2-leg ladder, Eq. (1.2) with $U / t$ small. It is then far from obvious that this analysis will apply to the infinite coupling limit, corresponding to the $t-J$ model of Eq. (1.2) so that comparisons with numerical results is important. In the weak coupling limit we may start by diagonalizing the non-interacting problem, giving symmetric and antisymmetric electron operators $\psi_{\lambda \alpha}$ where $\lambda=e$, o labels even and odd channels. We pass to the continuum limit by introducing left and right moving fields:

$$
\psi_{\lambda \alpha}(x)=e^{-i k_{F \lambda} x} \psi_{L \lambda \alpha}(x)+e^{i k_{F \lambda} x} \psi_{R \lambda \alpha}(x)
$$

with $k_{F e}$ and $k_{F o}$ the fermi wave vectors for the two bands. In the usual way, we represent the left and right moving fermion fields by left and right moving boson fields:

$$
\psi_{L / R \lambda \alpha} \propto e^{i \sqrt{4 \pi} \phi_{L / R \lambda \alpha}}
$$

and then, introducing the dual canonical Bose fields,

$$
\phi_{\lambda \alpha}=\phi_{R \lambda \alpha}+\phi_{L \lambda \alpha} \quad \theta_{\lambda \alpha}=\phi_{R \lambda \alpha}-\phi_{L \lambda \alpha}
$$

It is then convenient to introduce spin and charge bosons, for each channel:

$$
\begin{aligned}
& \phi_{\lambda \rho}=\left(\phi_{\lambda \uparrow}+\phi_{\lambda \downarrow}\right) / \sqrt{2} \\
& \phi_{\lambda \sigma}=\left(\phi_{\lambda \uparrow}-\phi_{\lambda \downarrow}\right) / \sqrt{2}
\end{aligned}
$$

and then, finally, switch to the two linear combinations of the even and odd bosons:

$$
\phi_{ \pm \rho}=\left(\phi_{e \rho} \pm \phi_{o \rho}\right) / \sqrt{2}
$$

with similar relations for $\phi_{ \pm \sigma}, \theta_{ \pm \rho}$, and $\theta_{ \pm \sigma}$. Actually, this last transformation is not canonical when the even and odd bosons have different relocities. However, we will follow the standard practice 13 of assuming that this velocity difference is irrelevant.

A renormalization group analysis, based on the weak coupling Hubbard model, suggests that the cosine interactions "pin" the bosons $\theta_{ \pm \sigma}$ and $\phi_{-\rho}$, introducing excitation energy gaps for these bosons and leaving $\phi_{+\rho}$ as the only massless boson which thus describes the low energy excitations. All interactions involving $\phi_{+\rho}, \theta_{+\rho}$ are irrelelvant in this phase. The low energy effective Hamiltonian can be written:

$$
H-\mu N=\frac{v_{+\rho}}{2} \int d x\left[K_{+\rho} \Pi_{+\rho}^{2}+\frac{1}{K_{+\rho}}\left(\frac{d \theta_{+\rho}}{d x}\right)^{2}\right],
$$

where $\Pi_{+\rho}$ is the momentum density variable canonically conjugate to $\theta_{+\rho}, v_{+\rho}$ is the velocity of the corresponding gapless low energy excitations and the parameter $K_{+\rho}$ controls the correlation exponents. (Our definition of $K_{+\rho}$ corresponds to that of Schuld 2 and Hayward and Poilbland but is the inverse of the parameter with the same name in Balents and Fisher.3) The Hamiltonian may be equally well written in terms of the other boson field $\phi_{+\rho}$ and its conjugate momentum using:

$$
\Pi_{\theta}=d \phi / d x, \quad \Pi_{\phi}=-d \theta / d x .
$$

The two parameters, $v_{+\rho}$ and $K_{+\rho}$ are generally difficult to calculate analytically and are extracted from numerical data. The long-range behaviors of various correlation functions are calculated straightforwardly by expressing the corresponding fermionic operators in terms of the bosons $\phi_{ \pm \sigma}, \phi_{ \pm \rho}$ and their duals. Exponentials of the pinned bosons can be replaced by their groundstate expectation values but exponentials of the duals of pinned bosons lead to exponentially decaying factors in correlation functions. Exponentials of the gapless boson, $\theta_{+\rho}$ and its dual give power law decaying factors. For instance, to calculate the uniform part of the pair correlation function we bosonize the pair operator:

$$
\begin{aligned}
\Delta_{e} & \equiv \psi_{L e \uparrow} \psi_{R e \downarrow} \propto e^{i \sqrt{4 \pi}\left(\phi_{L e \uparrow}+\phi_{R e \downarrow}\right)} \\
& \propto e^{i \sqrt{\pi}\left(\phi_{+\rho}+\phi_{-\rho}-\theta_{+\sigma}-\theta_{-\sigma}\right)} .
\end{aligned}
$$

We may replace the exponentials of $\phi_{-\rho}$ and $\theta_{ \pm \sigma}$ by a constant factor leaving simply the operator $e^{i \sqrt{\pi} \phi_{+\rho}}$. The correlation function for this operator decays as $1 /|x|^{1 /\left(2 K_{+\rho}\right)}$. The same result is obtained for the correlation function of a pair of electrons in the odd channel, $\Delta_{0}$. On the other hand, the correlation function $<\Delta_{e}^{\dagger}(x) \Delta_{o}(y)>$ is the same except for a factor of $<e^{i \sqrt{4 \pi} \phi_{-\rho}}>$. This is expected to be $<0$, based on the sign of the cosine interaction which pins the $\phi_{-\rho}$ boson to $\sqrt{\pi / 4}$, so this pair correlation function has the opposite sign, corresponding to "d-wave pairing". The $2 k_{F}$ part of the (even, spin-up) density operator is:

$$
e^{-2 i k_{F e} x} \psi_{R e \uparrow}^{\dagger} \psi_{L e \uparrow} \propto e^{-2 i k_{F e} x} e^{-i \sqrt{\pi}\left(\theta_{+\rho}+\theta_{-\rho}+\theta_{+\sigma}+\theta_{-\sigma}\right)} .
$$


The correlation function of this operator has exponential decay due to the $e^{-i \sqrt{\pi} \theta_{-\rho}}$ factor. Exponential decay is also obtained for all other terms in the $2 k_{F}$ part of the density operator. On the other hand, if we consider the correlation function for the square of the density operator, we get power-law decay for the $4 k_{F}$ part. This arises from terms of the form:

$$
\begin{array}{r}
e^{-2 i\left(k_{F e}+k_{F o}\right) x} \psi_{R e \uparrow}^{\dagger} \psi_{L e \uparrow} \psi_{R o \uparrow}^{\dagger} \psi_{L o \uparrow} \propto \\
e^{-2 i\left(k_{F e}+k_{F o}\right) x} e^{-2 i \sqrt{\pi}\left(\theta_{+\rho}+\theta_{+\sigma}\right)}
\end{array}
$$

We may replace $\exp \left(-2 i \sqrt{\pi} \theta_{+\sigma}\right)$ by its expectation value leaving only the gapless $\theta_{+\rho}$ field which gives power law decay:

$$
<n(x)^{2} n(0)^{2}>\propto \frac{\cos \left[2\left(k_{F e}+k_{F o}\right) x+\alpha\right]}{|x|^{2 K_{+\rho}}} .
$$

In fact we expect that the density operator itself will pick up a $4 k_{F}$ term proportional to this and also exhibit the same type of $4 k_{F}$ power law decay. This phase with one gapless charge mode and no gapless spin modes, labeled "C1S0" is expected to be the generic phase in the $2 \mathrm{leg}$ $t-J$ or Hubbard ladder. Whether this phase is better thought of as almost a d-wave superconductor, or almost an incommensurate CDW depends on the magnitude of $K_{+\rho}$. The pairing correlation function decays less rapidly than the density correlation function in the case $K_{+\rho}>$ $1 / 2$. Schulz has shown 11 that $K_{+\rho} \rightarrow 1$ as the density, $n$, approaches 1 . At $n=1$, the ladder is a spin gapped Mott insulator.

Thus it is of interest to calculate $K_{+\rho}$. This can be done from the finite size spectrum using either periodic or open boundary conditions. The latter are often used with DMRG so we discuss both cases. While, as we discuss below, it is enough to measure the excitation energies of only 3 states to extract $K_{+\rho}$ (and the velocity $v_{+\rho}$ ) we give formulas for an infinite number of low lying excitations. This allows for a more extensive check of the bosonization results with DMRG. Due to the somewhat conjectural nature of the extrapolation of weak coupling Hubbard results to the $t-J$ model this would be a worthwhile check. We discuss only the groundstate and lowlying excitations, whose excitation energies all scale to 0 as $1 / L$, where $L$ is the length of the system. These can be simply calculated from the free boson Hamiltonian of Eq. 2.6 when proper account is taken of the boundary conditions on the boson field, $\theta_{+\rho}$. We will generally set $v_{+\rho}=1$, restoring it by dimensional analysis when needed.

We first consider the case of periodic boundary conditions (b.c.'s). Clearly periodic b.c.'s on the fermions translate into periodic b.c.'s on the boson fields but we must take into account the fact that the boson fields are actually phase fields (only their exponentials and derivatives occur as physical local operators). Since all physical operators in the low energy effective theory involve integer powers of $\exp \left[i \sqrt{\pi} \phi_{+\rho}\right]$ and $\exp \left[i 2 \sqrt{\pi} \theta_{+\rho}\right]$, as in Eqs. (2.8) and 2.10), we see that:

$$
\begin{aligned}
\phi_{+\rho}(L) & =\phi(0)+2 \sqrt{\pi} m \\
\theta_{+\rho}(L) & =\theta(0)+\sqrt{\pi} p,
\end{aligned}
$$

where $m$ and $p$ are arbitrary integers. To obtain the complete low energy spectrum we simply write a mode expansion for the boson fields, consistent with these b.c.'s and the canonical commutation relations. This gives:

$$
\begin{aligned}
& \phi_{+\rho}(x)=\phi_{0}+\frac{2 \sqrt{\pi} m x}{L} \\
& +\sum_{k=1}^{\infty} \sqrt{\frac{1}{K_{+\rho} 4 \pi k}}\left(a_{R k} e^{i 2 \pi k x / L}+a_{L k} e^{-i 2 \pi k x / L}+\text { h.c. }\right) \\
& \theta_{+\rho}(x)=\theta_{0}+\frac{\sqrt{\pi} p x}{L} \\
& +\sum_{k=1}^{\infty} \sqrt{\frac{K_{+\rho}}{4 \pi k}}\left(a_{R k} e^{i 2 \pi k x / L}-a_{L k} e^{-i 2 \pi k x / L}+\text { h.c. }\right) .
\end{aligned}
$$

Here the quantum numbers $m$ and $p$ are integer valued. Using Eq. 2.7 we see that the zero wave-vector mode of the conjugate momentum, $\Pi_{\theta}$, has eigenvalues $2 \sqrt{\pi} m$. Thus the wave-functions for the zero-momentum modes are $\exp \left[i 2 \sqrt{\pi} m \theta_{0}\right]$. These wave-functions are invariant under $\theta_{0} \rightarrow \theta_{0}+\sqrt{\pi}$, as required by the angular nature of $\theta$. (The same result holds for the dual zero wave vector field, $\phi_{0}$ and its conjugate momentum.) The $a_{L K}$ and $a_{R k}$ operators annihilate left and right moving harmonic boson modes. We may give a physical interpretation to the quantum number $m$ using the bosonization formula for the total charge density:

$$
\begin{aligned}
J \equiv & \sum_{\lambda, \alpha}\left(\psi_{L, \lambda, \alpha}^{\dagger} \psi_{L, \lambda, \alpha}+\psi_{R, \lambda, \alpha}^{\dagger} \psi_{R, \lambda, \alpha}\right) \\
& \rightarrow(-1 / \sqrt{\pi}) \sum_{\lambda, \alpha} d \theta_{\lambda, \alpha} / d x \\
= & (-2 / \sqrt{\pi}) d \theta_{+\rho} / d x
\end{aligned}
$$

Thus the total charge, relative to that of the groundstate, is:

$$
Q=(-2 / \sqrt{\pi}) \int_{0}^{L} d x d \theta_{+\rho} / d x=-2 \Delta \theta_{+\rho} / \sqrt{\pi}=-2 p .
$$

Only excitations of even charge occur in the C1S0 phase. The other quantum number, $m$, measures the "chiral charge" which has a less obvious physical interpretation as the difference of charges of left and right movers.

Inserting Eq. (2.14) into the Hamiltonian of Eq. (2.6) and using the identity, Eq. (2.7) we can immediately read off the spectrum: 


$$
E-E_{0}=-2 p \mu+\frac{2 \pi v_{+\rho}}{L}\left[K_{+\rho} m^{2}+\frac{p^{2}}{4 K_{+\rho}}+\sum_{k=1}^{\infty} k\left(n_{L k}+n_{R k}\right)\right] .
$$

Here $n_{L k}$ and $n_{R k}$ are the occupation numbers for the left and right moving states of momentum $\pm 2 \pi k / L$. $E_{0}$ is the groundstate energy for a given density, $n$, and is non-universal. This formula gives the excitation energy for low-lying excitations with all quantum numbers $\ll L$. The parameters $\mu$ (chemical potential) $v_{+\rho}$ and $K_{+\rho}$ all depend on density.

The parameters $K_{+\rho}$ and $v_{+\rho}$ can be determined by measuring the excitation energies of three states. Generally one calculates the excitation energies of the states with $-\Delta Q / 2=p= \pm 1$ (and all other quantum numbers set to zero) to determine the ratio $v_{+\rho} / K_{+\rho}$, using:

$$
E(p=1)+E(p=-1)-2 E_{0}=\pi v_{+\rho} /\left(K_{+\rho} L\right) .
$$

The compressibility for a 2-leg ladder is generally defined as

$$
\frac{1}{n^{2} \kappa} \equiv \frac{1}{2 L} \frac{d^{2} E}{d n^{2}}
$$

where $n$ is the density. From Eqs. (2.15) and (2.16), this has the value $\pi v_{+\rho} /\left(2 K_{+\rho}\right)$. The velocity may be measured separately from the excitation energy of the lowest state of momentum $2 \pi / L$ :

$$
E\left(n_{R 1}=1\right)-E_{0}=\frac{2 \pi v_{+\rho}}{L} .
$$

Thus measuring three excitation energies, for fixed, large $L$, allows a determination of the correlation exponents. Using a large $L$ is important because corrections to Eq. (2.16) are only down by additional powers of $1 / L$. These corrections become especially large at commensurate filling near a transition to a CDW. The corresponding exponent will be given in Sec. III. While these three measurements are enough to determined the critical exponents, measurements of energies of additional states and a study of the $L$-dependence provide additional confirmation of the predictions of the RG and bosonization.

As mentioned above, DMRG works much more efficiently with open boundary conditions. We now discuss the finite size spectrum in that case. If we number the sites from 1 to $\mathrm{L}$ then we have a free boundary condition at $j=1$ and $j=L$. A free boundary condition on the fermion field at $j=1$ is equivalent to a vanishing boundary condition at $j=0$. [This follows from adding an extra "phantom site" at $j=0$ with associated hopping and exchange terms but then making these terms vanish by imposing $\psi_{\lambda \alpha}(0)=0$.] In terms of left and right movers this free boundary condition becomes:

$$
\psi_{L \lambda \alpha}(0)=-\psi_{R \lambda \alpha}(0) .
$$

Upon bosonizing we obtain:

$$
e^{i \phi_{L \lambda \alpha}}=-e^{i \phi_{R \lambda \alpha}}
$$

or

$$
\theta_{\lambda \alpha}(0)=\text { constant }
$$

(A determination of this constant involves consideration of some subtle commutators. We will not bother to keep track of it in what follows.) Clearly this boundary condition remains the same when expressed in the eventual basis $\rho / \sigma,+/-$. The only important boundary condition for the low energy excitations is:

$$
\theta_{+\rho}(0)=\text { constant }
$$

The same b.c. is obtained at $x=L+1 \approx L$ except that the constant will generally be different. Taking into account the periodic nature of $\theta$, Eq. (2.12), and the fact that $\Pi_{\theta} \propto \partial \theta / \partial t \propto \partial \phi / \partial x$ must also vanish at the boundaries, we see that the mode expansions become:

$$
\begin{aligned}
\phi_{+\rho}(x) & =\phi_{0}+\sum_{k=1}^{\infty} \sqrt{\frac{1}{K_{+\rho} \pi k}} \cos \left(\frac{\pi k x}{L}\right)\left(a_{k}+a_{k}^{\dagger}\right) \\
\theta_{+\rho}(x) & =\theta_{0}+\frac{\sqrt{\pi}(p-\alpha / 2 \pi) x}{L} \\
& +\sum_{k=1}^{\infty} \sqrt{\frac{K_{+\rho}}{\pi k}} i \sin \left(\frac{\pi k x}{L}\right)\left(a_{k}-a_{k}^{\dagger}\right) \cdot \quad
\end{aligned}
$$

Here $\alpha$ is proportional to the difference of constants appearing in the b.c.'s at $x=L$ and $x=0$. Substituting into the Hamiltonian, we now obtain the finite size spectrum:

$$
E-E_{0}=-2 p \mu+\frac{\pi v_{+\rho}}{L}\left[\frac{(p-\alpha / 2 \pi)^{2}}{2 K_{+\rho}}+\sum_{k=1}^{\infty} k n_{k}\right](2
$$

We see that Eq. 2.17) for $v_{+\rho} / K_{+\rho}$ remains true with free b.c.'s as we might expect due to the relation with the compressibility. We may now determine the velocity independently (and hence determine $K_{+\rho}$ ) by measuring the gap to the first excited state with the same charge as the groundstate:

$$
E\left(n_{1}=1\right)-E_{0}=\frac{\pi v_{+\rho}}{L} .
$$

Note that this gap has half the value of the gap to the lowest energy state of momentum $2 \pi / L$ in the case of periodic b.c.'s, Eq. (2.19). This is just a consequence of 
the familiar result that the spacing of wave-vectors for open b.c.'s is $\pi / L$, half the spacing for periodic b.c.'s.

Previous measurements of the parameter $K_{+\rho}$, and its analogue in other chain and ladder systems, have used periodic boundary conditions. Exact diagonalization, or "modified Lanczos" methods work efficiently to determined the lowest energy state of given quantum numbers. Thus the energy differences of Eqs. (2.17) and (2.19) can be measured from finding the lowest energy states with various charges and with momenta 0 and $2 \pi / L$. An alternative approach is to meaure the dependence of the groundstate energy (for a fixed charge) on an applied flux, i.e. a twist in the boundary conditions on the fermion fields:

$$
\psi_{L / R \lambda \alpha}(L)=e^{i \Phi} \psi_{L / R \lambda \alpha}(0) .
$$

This corresponds to putting a twist into the boundary condition on $\phi_{+\rho}$ in Eq. (2.12), as can be seen from the fact that all the fermion fields ( $L$ or $R$ ) contain a phase factor of $\exp \left[i \sqrt{\pi} \phi_{+\rho} / 2\right]$.

$$
\phi_{+\rho}(L)=\phi_{+\rho}(0)+2 \sqrt{\pi}(m+2 \Phi) .
$$

From Eq. (2.16) we see that the groundstate energy is increased by:

$$
E_{0} \rightarrow E_{0}+8 \pi v_{+\rho} K_{+\rho} \Phi^{2} / L .
$$

Hence measuring the flux dependence of the groundstate energy determines $v_{+\rho} K_{+\rho}$, while measuring the compressibility determines $v_{+\rho} / K_{+\rho}$. Hayward and Poilbland measured $K_{+\rho}$ in this way for the 2-leg ladder using a maximum system size of $2 \times 10$. Another approach was taken by Siller et al. 1 They modeled the 2-leg tJ model as a single chain model of bosonic hole pairs. The parameters in the effective Hamiltonian for hole pairs were determined from DMRG calculations with open boundary conditions on system sizes up to $40 \times 2$ for systems containing 2 holes or 4 holes only. The resulting bosonic model was then studied using exact diagonalization (Lanczos) methods and periodic boundary conditions. Since the number of effective bosons (of density $x / 2$ where $x \equiv 1-n$ ) is small near $1 / 2$-filling, it was possible to diagonalize large systems (up to 220 sites in the case of only 2 bosons).

The approach advocated here, working exclusively with open boundary conditions, has been used previously for spin chains 14 but not, as far we we know, for tJ ladders. It allows us, using DMRG, to study much larger system sizes, up to $192 \times 2$, than are accessible with Lanczos in the usual formulation. It furthermore avoids making any assumptions which are necessary in treating the hole pairs as bosons, such as the particular form of the boson-boson interaction and the absence of three-boson terms, which could induce some density dependence in the interaction. However, the DMRG calculations with the accuracy and system length required to determine $K_{+\rho}$ for these ladder systems were surprisingly difficult. In order to minimize the effect of correction terms to the asymptotic formulas, long systems were needed. In these systems, one is probing competing pairing and charge fluctuations at very low energies and large distances, which is difficult in DMRG. Typically more than 2000 states were kept in the calculations and more than a dozen sweeps were performed. Some of the calculations were more accurate and reliable than others, however. In Fig. 11 we show a plot of the left-hand side of Eq. 2.17) versus $1 / L$ for three values of $(J, n)$, showing linear behavior and allowing us to extract $v_{+\rho} / K_{+\rho}$. These calculations require only the ground state energy, for which an extrapolation in the energy versus the truncation error is very reliable. Thus this determination of $v_{+\rho} / K_{+\rho}$ is the most reliable and accurate ingredient in the determination of $K_{+\rho}$, and, in fact, the results shown could be improved with modest additonal effort.

In Fig. 2 we plot the left-hand side of Eq. (2.26) versus $1 / L$, again obtaining linear behavior. These calculations were difficult. It is necessary to target two states simultaneously, and extrapolation is not very useful. Note that, if $L$ is not large enough and the (infinite $L$ ) spin gap is not small enough, $\Delta_{s}<2 \pi v_{+\rho} / L$, then the first excited state with the same quantum numbers might actually have $S=1, S^{z}=0$ and correspond to a spin excitation, rather than the desired neutral excitation of the $+\rho$ field. (It might also be a neutral excitation of one of the other gapped boson fields.) In order to check the former possibility, we have calculated the spin gap, i.e. the excitation energy for the lowest state with $S^{z}=1$. We find that the very nonlinear behavior for $J=0.35, n=0.75$ shown in Fig. 2 is due to a small spin gap in this system, of order 0.04 . Thus it was necessary to study a $96 \times 2$ system, for which we kept 4000 states, and performed a dozen iterations, to clearly see the required gap. We be-

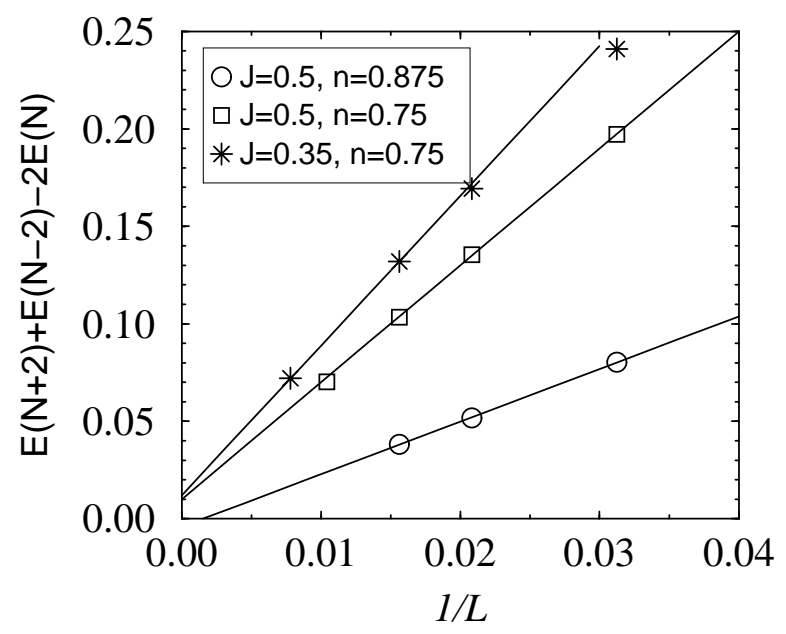

FIG. 1: These results were obtained by targetting three different ground states with varying numbers of particles. The straight lines are linear fits to the data. The slopes of the fits (which asymptotically yield $\pi v / K$ ) are 2.70 for $\mathrm{J}=.5$, $\mathrm{n}=0.875,6.75$ for $\mathrm{J}=.5, \mathrm{n}=0.75$ and 7.68 for $\mathrm{J}=.35, \mathrm{n}=0.75$. 


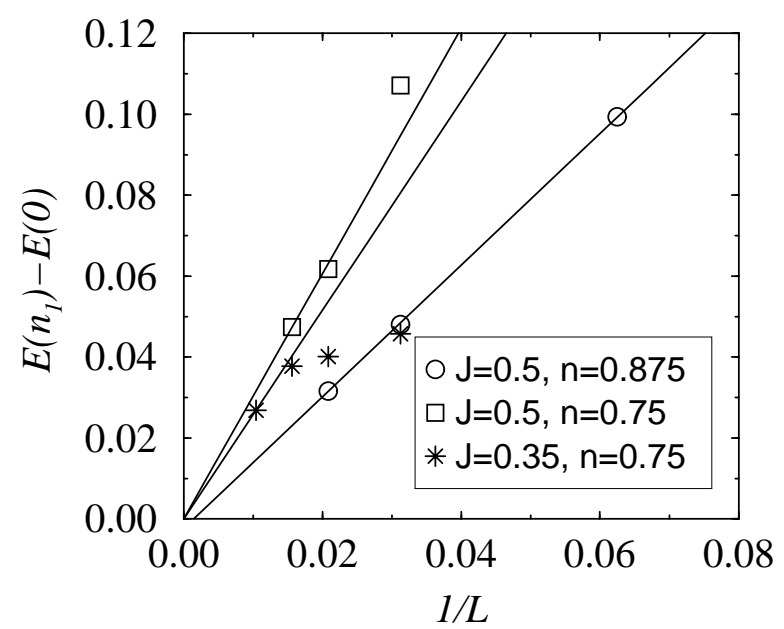

FIG. 2: Excitation energy for lowest state with $S^{z}=0$ and same electron density. The spin gap is about 0.14 for $\mathrm{J}=0.5$, $\mathrm{n}=0.875$, about 0.10 for $\mathrm{J}=.5, \mathrm{n}=0.75$ and about 0.07 for $\mathrm{J}=.35, \mathrm{n}=0.75$. The breakdown of the asymptotic linear behavior is clearly visible in the third case, at energies of order the spin gap. The straight lines are linear fits to the data. The slopes of the fits (which asymptotically yield $\pi v$ ) are 1.631 for $\mathrm{n}=0.875, \mathrm{~J}=0.5 ; 3.03$ for $\mathrm{n}=0.75, \mathrm{~J}=0.5$; and 1.6368 for $\mathrm{n}=0.75, \mathrm{~J}=0.35$.

\begin{tabular}{r|r|r|r}
$(n, J)$ & $v$ & $K_{\text {energy }}$ & $K_{\text {amp }}$ \\
\hline$(0.875,0.5)$ & 0.519 & 0.604 & 0.633 \\
$(0.75,0.5)$ & 0.964 & 0.449 & 0.359 \\
$(0.75,0.35)$ & 0.796 & 0.33 & 0.284
\end{tabular}

TABLE I: Results for the charge velocity $v$ and Luttinger liquid parameter $K$. $K_{\text {energy }}$ comes from using Eqs. (2.17) and (2.26), while $K_{a m p}$ is determined using the decay of the Friedel oscilations in the center of the system as a function of the system length.

lieve this data is reliable, but due to the large numerical work required we have only studied three different values of $(J, n)$. Combining the results of Figs. 1 and 2 allows a determination of $v_{+\rho}$ and hence $K_{+\rho}$. The resulting values are given in Table II.

\section{CHARGE DENSITY WAVES}

While a C1S0 phase is expected over most of the parameter range in the 2-leg $t-J$ model, a completely gapped, charge density wave (CDW) phase may occur at special commensurate filling factors, depending on the value of $J / t$ (and other possible interaction parameters). 12.13 In this section we review the conditions on the critical exponent parameter, $K_{+\rho}$ for a CDW to occur and discuss numerical work on the $t-J$ model at electron densities $n=3 / 4$ and $n=1 / 2$, corresponding to fillings of $3 / 8$ and $1 / 4$ respectively.
The low energy effective Hamiltonian only contains Fourier modes of the electron fields within a small momentum range, $\pm \Lambda$ of $\pm k_{F}$. Umklapp type interaction terms, which do not conserve separately the number of left and right movers, are generally accompanied by rapidly oscillating phase factors. They therefore do not appear in the low energy effective Hamiltonian since the electron fields vary slowly and hence these rapidly oscillating factors cause these interactions to average to zero. However, at special filling factors, corresponding to special values of the Fermi wave-vector, the oscillating factors become constants and these operators then appear in the effective Hamiltonian. Whether or not they produce a gap and a CDW depends on whether or not they are relevant operators in the RG sense. In a 1-dimensional relativistic quantum field theory an operator is relevant if it has a scaling dimension, $x<2$. This scaling dimension determines the exponent, $\eta$ with which the correlation function of this operator decays, with:

$$
\eta=2 x .
$$

Using the free boson Hamiltonian of Eq. (2.6), the scaling dimension of any operator is easily obtained. For example, the Hubbard or $t J$ interaction leads to an interaction term of the form given in Eq. (2.10). We see that this oscillates at wave-vector $2\left(k_{F e}+k_{F o}\right)$. Only when this wave-vector is a mutiple of $2 \pi$ will this operator appear in the low energy effective Hamiltonian. Furthermore, any operators whose correlation functions decay exponentially are irrelevant.

The number of electrons, $N_{\lambda}$ in each band is:

$$
N_{\lambda} / L=2 k_{F \lambda} / \pi
$$

(The factor of 2 arises from spin.) Thus:

$$
2\left(k_{F e}+k_{F o}\right)=2 \pi n,
$$

where:

$$
n \equiv\left(N_{e}+N_{o}\right) / 2 L,
$$

is the electron density. While the value of $k_{F e}$ and $k_{F o}$ may be renormalized by interactions (and may, in fact, not really be well-defined in the interacting model) their sum is "protected" by the one-dimensional version of Luttinger's theorem 15 so that Eq. (3.3) is expected to be exact.

Thus we see that the Umklapp operator of Eq. (2.10) can only occur in $H_{\text {eff }}$ for $n=1$ (half-filling). This operator has scaling dimension:

$$
x=K_{+\rho} .
$$

At $1 / 4$ filling, corresponding to $n=1 / 2$, the product of this operator times itself with spin up replaced by spin down, can occur, giving rise to an operator containing 8 electron operators. Under bosonization this is proportional to $\exp \left[-4 i \sqrt{\pi} \theta_{+\rho}\right]$ and has dimension $x=4 K_{+\rho}$. 
At a filling of $3 / 8$ (or $1 / 8$ ) corresponding to $n=3 / 4$ (or $1 / 4$ ) the fourth power of the basic Umklapp operator in Eq. (2.10) containing 16 electron operators can occur. It has dimension $x=16 K_{+\rho}$.

The condition on the RG scaling dimension, $x$, for an operator to induce a CDW is somewhat subtle, and depends on whether the density or $J / t$ is varied. If we vary $J / t$, holding the density fixed at the commensurate value, then the system will remain in the gapless C1S0 phase as long as $x>2$, so that the operator is irrelevant. A transition to a gapped CDW phase occurs at the value of $J / t$ where $x$ becomes $<2$. In the CDW phase the value of $K_{+\rho}$ becomes undefined since all correlation functions decay exponentially (or go to constants). We may also consider what happens as we vary the density in the vicinity of the commensurate value. If the system is not in the CDW phase at a commensurate filling, then we expect $x$ to vary smoothly and, of course, to satisfy $x>2$ at the commensurate point. On the other hand, if the system is in the CDW phase at a commensurate filling, then we expect $x \rightarrow 1$ as the commensurate filling is approached. This follows from a general and clever argument by Schulz 22 based on "refermionizing" the single boson Hamiltonian. The relevant operator always behaves like a fermion mass term, of scaling dimension $x=1$ very close to the commensurate filling, whenever a CDW occurs at that point. It follows that, $x$ must vary rapidly in the vicinity of the critical value of $J / t$ where the CDW transition occurs near a commensurate filling. On the CDW side of the transition $x$ will be close to 2 away from the commensurate filling but then drop abruptly to 1 as that filling is approached. We emphasize that Schulz's argument, which was formulated in terms of the single boson sine-Gordon theory, is very general and doesn't depend on the underlying microscopic model. Similar behavior can occur for a $t-J$ or Hubbard ladder with any number of legs. Such rapid variation of $K$ in the vicinity of commensurate filling for a system with a CDW was observed by Schulz for the single leg Hubbard model near half-filling, by obtaining $K$ from the Bethe ansatz and similar behavior was also observed near $1 / 2$-filling $(n=1)$ for the 2-leg $t-J$ model by Siller et al.

Now consider a density $n=1 / 2$ corresponding to $1 / 4$ filling. If we sit at this density and vary $J / t$ then a CDW transition occurs when $K_{+\rho}=1 / 2$, with the gapless phase having $K_{+\rho}>1 / 2$. On the other hand, if $J / t$ is such that the system is in a CDW phase at $n=1 / 2$, then $K_{+\rho} \rightarrow 1 / 4$ as $n \rightarrow 1 / 2$.

Similarly, a CDW at $3 / 8$ filling, $n=3 / 4$, is signalled by $K_{+\rho} \rightarrow 1 / 8$, as we vary $J / t$ at fixed $n$ or $K_{+\rho} \rightarrow 1 / 16$ as we vary the density at fixed $J / t$ in the CDW phase. See Fig. 3 .

The connection between the relevance of the multiple Umklapp interaction and the presence of a CDW is easily established. When the multiple Umklapp term (for $n=1 / 2$ or $3 / 4)$ is relevant it pins the $\theta_{+\rho}$ boson. From Eqs. (2.10) and (3.3) we see that the $4 k_{F}$ term in the density operator then has a non-zero groundstate expec-

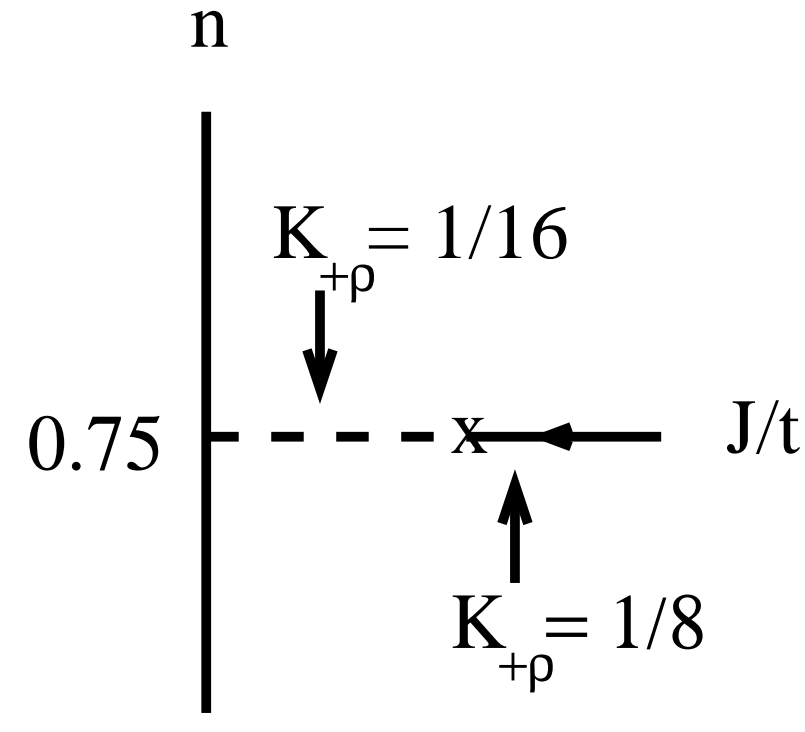

FIG. 3: The qualitative behavior of $K_{+\rho}$ as a function of $J / t$ and $n$. A CDW occurs along the dashed line at $n=.75$ so $K_{+\rho}$ is not well-defined there. As this line is approached by varying $n, K_{+\rho} \rightarrow 1 / 16$. On the other hand, at $n=.75$ and larger $J / t$ there is no CDW and $K_{+\rho}$ is well defined, with a value approaching $1 / 8$ at the CDW critical point.

tation value, so that

$$
<n_{j}>\propto \cos (2 \pi n j+\alpha)+\text { constant },
$$

where $\alpha$ is a constant. Thus the density oscillations should have wavelength 2 for $n=1 / 2$ and wavelength 4 for $n=3 / 4$. In general, it is sometimes convenient to introduce

$$
\delta \equiv 1-n,
$$

the density of holes measured from half-filling, sometimes called the density of "holons" and proportional to the doping parameter in the cuprates. We see that $n$ can be replaced by $\delta$ in Eq. (3.6) so that the wavelength of the CDW oscillations is $1 / \delta$. For a 2-leg ladder, this is the average horizontal separation of hole pairs. ("Horizontal" refers to the direction along the legs of the ladder.)

The CDW corresponds to a broken translational symmetry, so that there are two different groundstates for $\mathrm{n}=1 / 2$ and four different groundstates for $\mathrm{n}=3 / 4$ (differing only by translation by 1 site). For a finite ladder with periodic b.c.'s we expect quantum tunnelling between these groundstates to occur so that no density oscillations exist in the finite system groundstate. On the other hand, with open b.c.'s one or the other of the groundstates gets picked out by the boundary conditions so that the CDW is directly observable.

We now return to the point raised in Sec. II, the higher order corrections in $1 / L$ to the finite size energy gaps given in Eqs. (2.16) and (2.25). The leading correction is determined by the leading irrelevant operator. At a commensurate filling, when the system is 
close to having a CDW, the multiple Umklapp operator discussed above has a dimension $x$ only slightly greater than 2 , corresponding to being barely irrelevant. In this case the higher order terms in the energy formula are of $O\left(1 / L^{x-1}\right)$. This will make it difficult to determined $K_{+\rho}$ reliably from finite size gaps in the vicinity of a $\mathrm{CDW}$, complicating the determination of the CDW phase boundary.

Previously reported work $\mathrm{b}$ based on the bosonic hole pairs approximation together with DMRG and Lanczos, on the 2-leg $t J$ model, with $J / t=.35$, found a smooth behavior of $K_{+\rho}$ in the vicinity of $n=3 / 4$ with a value at a commensurate density $n=3 / 4$ of approximately .232. Using the direct DRMG approach, we have found $K_{+\rho} \approx .33$. Both results are far above the critical values $(1 / 8$ and $1 / 16)$, indicating that a CDW does not occur at $n=3 / 4$ for $J / t=.35$. The charge gap for $n=.75$ with $J=.35$ and $J=.25$ is plotted versus $1 / L$ in Fig. 4. There is, indeed, no evidence for a charge gap at the larger value of $J$, consistent with the absence of a CDW. On the other hand, there is evidence for a charge gap at $J=0.25$. Note that these calculations involve only ground state energies and thus are very reliable. Although the results are extrapolated to zero truncation error, the extrapolation is quite small in magnitude, of order the symbol size in the figure, and the estimated error in the extrapolation is very small, as shown by the error bars. These results provide some evidence that there is a criticial value of $J$ between 0.25 and 0.35 such that a charge gap and CDW occur for smaller $J$.

As reviewed in the next section, if there is no CDW, and the system in is the C1S0 phase, we expect the density oscillations at the center of the chain to decay with chain length as $L^{-K_{+\rho}}$ with $K_{+\rho}>1 / 8$. Comparing the oscillation amplitude for $n=.75, J=.25$ and the two longest lengths studied, $\mathrm{L}=96$ and $\mathrm{L}=192$, we find a ratio of oscillation amplitudes of .915 which would give an exponent of $K_{+\rho}=.128$. This is slightly larger than the critical value of $1 / 8$. On the other hand, it is so close to the critical value that we might wonder if the density oscillation amplitude would eventually approach a constant with still longer chain lengths corresponding to a true CDW.

Another important question is the behavior of the spin gap in the putative CDW phase. Since the Umklapp term which drives the CDW does not contain the spin bosons, it is natural to assume that the gapping of the charge boson, corresponding to the CDW, occurs without any major effects on the other bosons which could thus remain gapped. However, it is possible that one (or more) of the other bosons, such as a spin boson, becomes gapless at the same transition. Indeed, the occurence of the CDW may be related to the close proximity of a phase with no spin gap.

In Fig. 5 , we show the spin gap on a $16 \times 2$ system for a wide range of $J$. The data suggests that in the thermodynamic limit, the spin gap vanishes near $J=0.25$. To verify this result, one must perform a finite size study.

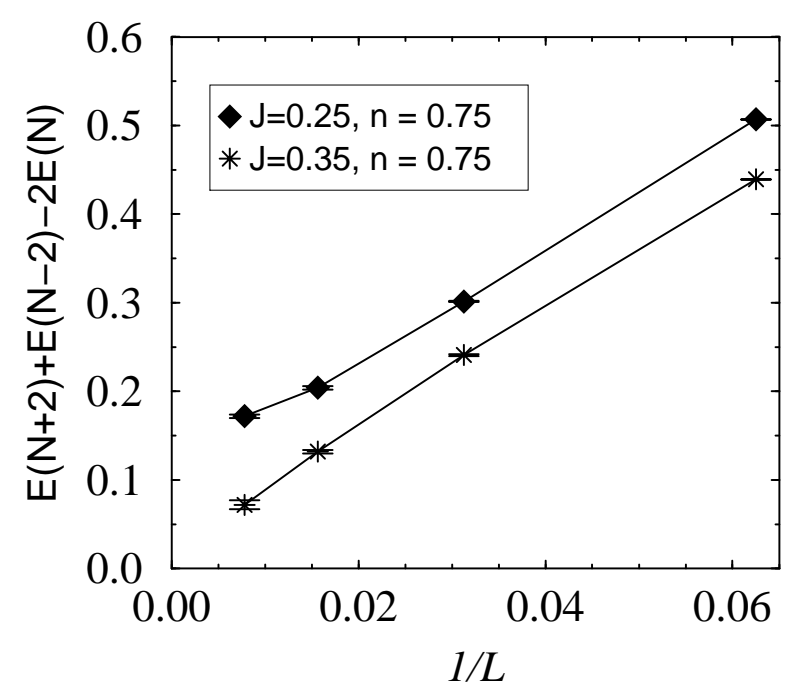

FIG. 4: Scaling of the charge gap with $1 / L$, showing the occurance of a CDW at $n=.75$ for $J=.25$ but not $J=.35$.

However, the spin density pattern on the $16 \times 2$ system for $J=0.2$ has the largest values of $\left\langle S_{i}^{z}\right\rangle$ concentrated near the ends of the system, indicating that an edge excitation has the lowest energy. In order to ensure that we have a bulk spin excitation in the calculation, we next studied systems where we increased the rung exchange interaction to $J+0.3$ on the first and last rungs of a ladder. This drives up the energy of the edge excitation, and one finds that the resulting spin pattern is concentrated in the central region of the ladder. In Fig. 6, we show the spin gap as a function of $1 / L$ for several systems. The results for $J=0.2, n=0.75$ are consistent with a gapless

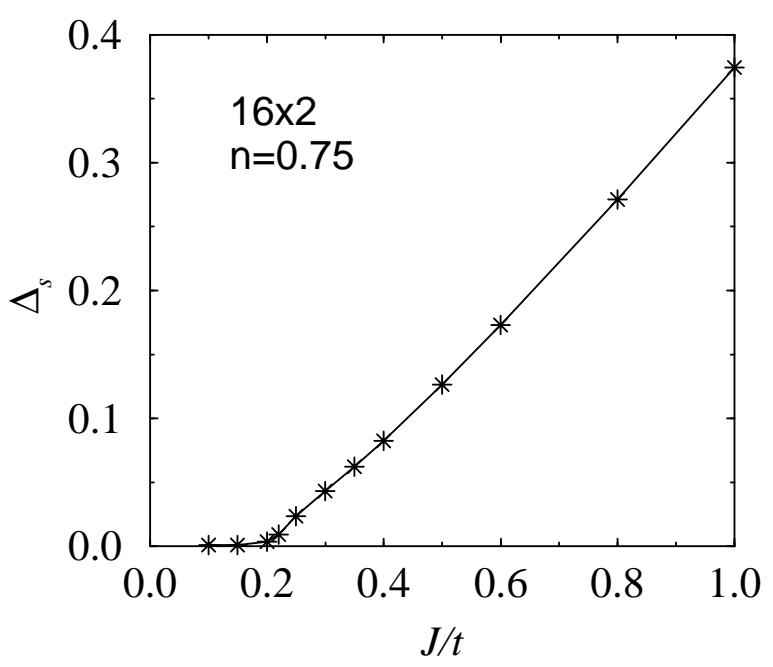

FIG. 5: Spin gap on a $16 \times 2$ system as a function of $J / t$. 


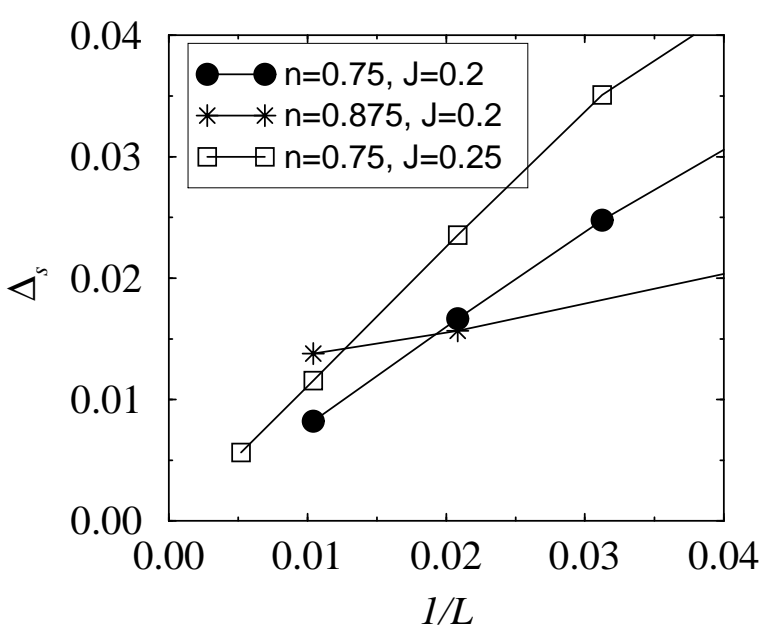

FIG. 6: Spin gap as a function of $1 / L$ for several systems. The values of the exchange on the first and last rungs have been altered to avoid edge excitation.

state, although one can never rule out a very small gap. Similarly, for $J=0.25, n=0.75$ no evidence for a spin gap on the largest system, a $192 \times 2$ ladder. For $J=0.2$, $n=0.875$ we see a clear spin gap of order 0.013 .

The proximity to a phase with vanishing spin gap may be related to the occurence of the CDW. As the spin gap gets smaller, and the corresponding length scale larger, we may expect the size of the 2-hole pairs to get larger. This is naturally associated with a growing scattering length for the effective boson system, which enhances the formation of a CDW.

A CDW with vanishing spin gap at $n=3 / 4$, can be easily understood heuristically. One can imagine one hole localized on every second rung, with the electrons on doubly occupied rungs forming spin singlets, as illustrated in Fig. 7, giving an effective $S=1 / 2$ Heisenberg chain with lattice spacing 2 which has vanishing spin gap. On the other hand, in the spin-gapped phase we can think of pairs of holes localized on every fourth rung, as in fig. 8 . Thus we expect the groundstate density oscillations to have wavelength 2 in the CDW with vanishing spin gap but wavelength 4 in the gapped CDW.

The connection between wavelength doubling and the spin gap is related to the Lieb-Schultz-Mattis theorem.15 If the wavelength is not doubled to 4 , then gapless excitations are expected. More precisely, we can prove the existence of gapless excitations at $n=3 / 4$ if the groundstate is invariant under translations by 2 and under site parity: $j \rightarrow-j$ and is not ferromagnetic. (Note that link parity, $j \rightarrow-j+1$ is spontaneously broken by the wavelength 2 groundstate illustrated in Fig. 7 but site parity is not.) This is proven by considering a long ladder of even length $L$ with periodic b.c.'s. Let $\mid \psi_{0}>$ be a groundstate. We then consider the variational state ob-

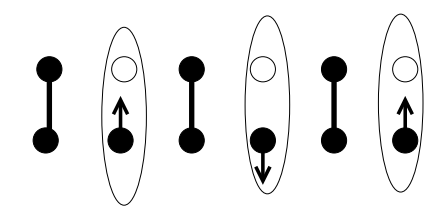

FIG. 7: A charge arrangement with period 2 at $n=3 / 4$, corresponding to a gapless CDW. The black circles represent electrons and the white circles holes. The ovals indicate that the location of the hole is symmetrized between the two sites on a rung. The solid lines indicate the formation of dimer singlets. The arrows indicate the spins of the unpaired electrons, but we donnot expect them to be Néel ordered in this one-dimensional system.

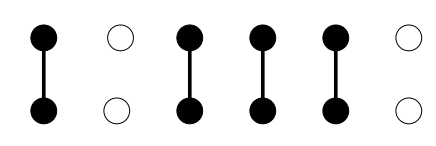

FIG. 8: A charge arrangement with period 4 at $n=3 / 4$, corresponding to a gapped CDW. (The actual density oscillations seem to correspond to pairs of holes shared evenly by every second pair of rungs.)

tained by acting on the $\mid \psi_{0}>$ with the unitary operator:

$$
U=\exp \left[i(2 \pi / L) \sum_{j} j n_{j \uparrow}\right]
$$

where $n_{j \uparrow}$ is the number operator for spin up electrons on rung $j$ (summing over both sites on the rung). It is straightforward to calculate $<\psi_{0}\left|U^{\dagger}\left(H-E_{0}\right)\right| \psi_{0}>$ and show that it is $O(1 / L)$ provided that the following term vanishes:

$$
\sum_{j}\left[<\psi_{0}\left|c_{j \lambda \uparrow}^{\dagger} c_{j+1 \lambda \uparrow}\right| \psi_{0}>-<\psi_{0}\left|c_{j+1 \lambda \uparrow}^{\dagger} c_{j \lambda \uparrow}\right| \psi_{0}>\right] .
$$

We can show that this vanishes, for example, by using the assume site parity symmetry to show that:

$$
<\psi_{0}\left|c_{j \lambda \uparrow}^{\dagger} c_{j+1 \lambda \uparrow}\right| \psi_{0}>=<\psi_{0}\left|c_{j \lambda \uparrow}^{\dagger} c_{j-1 \lambda \uparrow}\right| \psi_{0}>.
$$

To complete the proof of a low energy state we must prove that $U \mid \psi_{0}>$ does not become $\mid \psi_{0}>$ in the limit $L \rightarrow \infty>$. We prove this by considering the behavior of $U$ under translations by 2 sites, the assumed symmetry of the groundstate. We fine that under this translation:

$$
U \rightarrow U e^{i 8 \pi n_{\uparrow}},
$$

where $n_{\uparrow}$ is the density of spin up electrons, $n_{\uparrow}=N_{\uparrow} / 2 L$, where $N_{\uparrow}$ is the total number of spin up electrons and $2 L$ is the number sites on a 2-leg ladder of length $L$. If we further assume that $n_{\uparrow}=n_{\downarrow}$, i.e. that the groundstate is not ferromagnetic, then we may replace the exponential factor in Eq. (3.11) by $e^{i 4 \pi n}$. For a density, $n=3 / 4$, this factor is -1 . This proves orthogonality of $U \mid \psi_{0}>$ with $\mid \psi_{0}>$ and hence the existence of a low energy excitation. On the other hand, if the groundstate is only 
invariant under translations by 4 sites then, repeating the argument with a translation by 4 sites changes the exponential factor to $e^{i 8 \pi n}$ which is 1 for $n=3 / 4$. Thus we cannot prove that $U \mid \psi_{0}>$ is orthogonal to the groundstate in this case; it may approach it in the limit $L \rightarrow \infty$, so the proof collapses. Unfortunately, this does not prove that there is a gap when the groundstate has wavelength 4 , only that the gap neccessarily vanishes when it has wavelength 2 . However, it often appears to be the case that when the LSM theorem fails, a gap appears. for instance, for $\mathrm{S}=1 / 2$ spin chains, a gap is expected whenever the groundstate has wavelenth 2 , as in a dimerized state. Thus, the nature of the density oscillations provides additional evidence for which phase the system is in.

To see what Fourier components are present in the density oscillations, we Fourier transform the density as a function of rung position and plot the power spectrum. In order to avoid spurious edge effects, a windowing function is used. If the original density is $d(x)$, we Fourier transform $D(x)=W(x) d(x)$, where $W(x)$ is a smooth windowing function. $W(x)$ is chosen to vanish at $x=1$ and $x=L$, and to be unity for the middle third of the $x$ values, with a smooth continuation in between; the particular choice we use is given in Ref. (16). Before Fourier transforming, we calculate the sum $I$ of $D(x)$ from 1 to $L$, and the sum $J$ of $W(x)$, and then subtract from $D(x)$ the function $\frac{I}{J} W(x)$. This removes a large peak centered at $k=0$ which is uninteresting. The lattice spacing is set to 1 , so that the exponential in the FT is $\exp (-i k j)$, where $\mathrm{j}$ runs over integer lattice sites. With this approach there is no restriction on the allowed values of $k$; the finite value of $L$ instead leads to finite widths for the peaks.

The density oscillations for $J=.25$ and also $J=.2$, with $n=.75$ have a very strong component at a wavelength of 4 , corresponding to a spin-gap, as shown in figure 9 .

\section{A POSSIBLE PHASE DIAGRAM}

In this section we briefly discuss a possible phase diagram and the qualitative behavior of $K_{+\rho}$ as a function of $J$ and $n$ for the 2-leg $t-J$ model. We are interested in the region $n \geq 0.5$ where both the even and odd parity bands have carriers in the weak coupling Hubbard limit. At still lower doping the system is expected to enter a C1S1 phase. In the region $n \geq .5$ our phase diagram is similar to that proposed by Hayward and Poilblanc, and is based on their exact diagonalization results, earlier DMRG results, our new DMRG results and some general results which follow from bosonization and RG. This is sketched in Fig. 10. Here the two ordered CDW phases at $\mathrm{n}=0.75$ and $\mathrm{n}=0.5$ are indicated by solid cuts for small values of $\mathrm{J} / \mathrm{t}$ and a phase separation region occurs for large $\mathrm{J} / \mathrm{t}$ values. We conjecture that the remaining region is in a C1S0 (d-wave- $4 \mathrm{k}_{F} \mathrm{CDW}$ ) phase. As discussed in Sec. II, this phase has the $\phi_{-\rho}$ and $\theta_{ \pm \sigma}$ bosons pinned

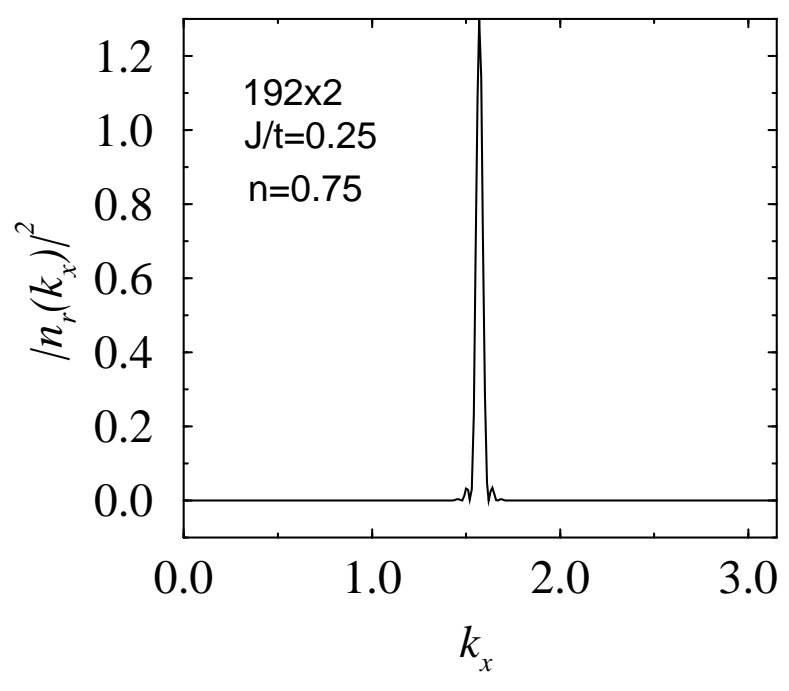

FIG. 9: Smoothed Fourier transform of the density oscillations showing only a large peak at wave-vector $\pi / 2$ corresponding to a wavelength of 4 .

and is characterized by power law d-wave pairing and $4 \mathrm{k}_{F}$ CDW correlations. When $K_{+\rho}>0.5$, the pairing correlations are dominant. Supporting this conjecture, DMRG calculations for $\mathrm{J} / \mathrm{t}$ and $\mathrm{n}$ values near the phase separation boundary show clear power law d-wave like pairing correlations.

The phase diagram shown in Fig. 10 for the $t-J$ ladder differs in several ways from that expected for the 2-leg repulsive U Hubbard model. Firstly, it is generally believed that the Hubbard ladder doesn't exhibit phase separation and the ordered CDW phases are absent. Secondly, weak coupling renormalization-group studies, bosonization [4] and DMRG calculations suggest that $K_{+\rho} \leq 1$ for the repulsive U Hubbard model. For an extended Hubbard model with additional interactions one can have $K_{+\rho}>1$; however Orignac and Giamarchil argue that when $K_{+\rho}>1$, the d-wave- $4 \mathrm{k}_{F}$ CDW sector gets replaced by a C1S0 s-wave pairing or an orbital antiferromagnetic phase. This raises questions regarding the phase diagram show in Fig. 10. We believe that while alternate C1S0 phases are certainly logical possibilities, they do not occur in the $t-J$ ladder. The argument that they occur whenever $K_{+\rho}>1$ appears to depend upon a weak coupling analysis which may not be applicable to the $t-J$ ladder. In this analysis, the phase boundaries between the various C1S0 phases is determined by the equality of 2 small marginal coupling constants in the Hamiltonian. These coupling constants also determine $K_{+\rho}$, in the weak coupling limit, in such a way that when they are equal, $K_{+\rho}=1$. [See Eq. (35) of Ref. (11).] For stronger coupling, and, in particular, for the $t-J$ model, the possible phase boundary to a phase with orbital antiferromagnetism need not be related in any general way to the value of $K_{+\rho}$ and the DMRG results suggest that or- 


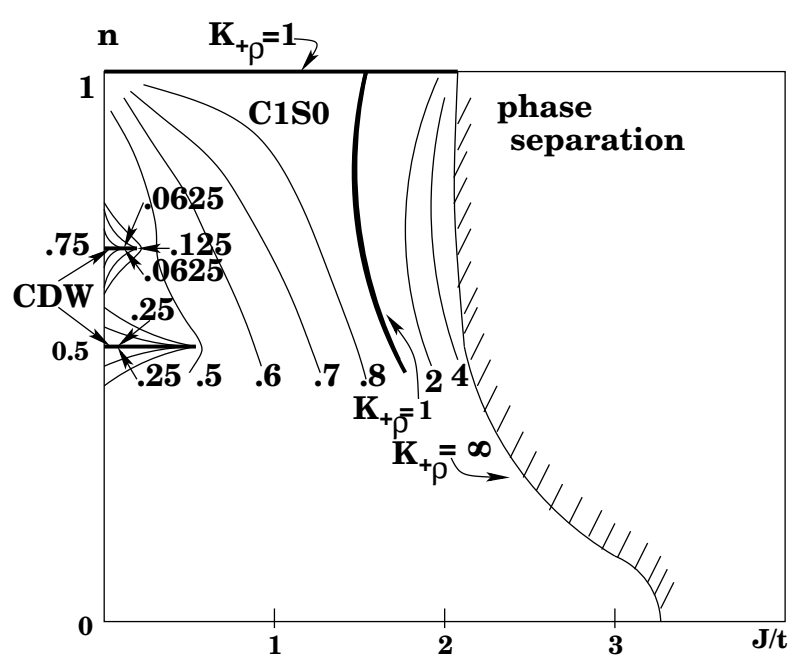

FIG. 10: Schematic phase diagram for the $t-J$ ladder as a function of $J / t$ and the electron density $n$ in the region $n$ greater than about .5 .

bital antiferromagnetism does not occur in the ordinary $t-J$ model.17

The phase separation boundary was obtained in a previous DRMG study 18 As this boundary is approached the compressibility diverges and $K_{+\rho} \rightarrow \infty$.

We have taken into account the behavior of $K_{+\rho}$ near commensurate fillings in drawing Fig. 10. In particular, at $n \rightarrow 1$, the $t-J$ model reduces to the 2-leg Heisenberg spin ladder and has an infinite gap for all charge excitations and presumably a gap of $O(J)$ for spin excitations. The general arguments of Schulz 211 then imply that $K_{+\rho} \rightarrow 1$ along the line $n=1$. We have also assumed that a CDW occurs at $n=.75$ and $n=.5$ for small enough $J$. As discussed in the previous section, we have found some evidence for such a phase at $J=.25$ and $\mathrm{n}=.75$. The corresponding behavior of $K_{+\rho}$, reviewed in the previous section is incorporated in Fig. 10. Note that, at small $J, K_{+\rho}$ apparently varies rapidly between .0625 for $n=.75$ and 1 for $n=1$. As we also discussed in the previous section, it is possible that the spin gap vanishes at commensurate fillings for some range of $J$. We do not attempt to indicate this possibility in Fig. 10.

For values of $J / t$ around .35, which has been argued to be a reasonable value for modeling the cuprates, $K_{+\rho}<.5$ near $\mathrm{n}=.75$ but increases towards 1 as $\mathrm{n}=1$ is approached. Thus there is only a narrow window of doping near $1 / 2$-filling where d-wave pairing correlations dominate.

\section{FRIEDEL OSCILLATIONS}

At incommensurate filling, or in general when no CDW occurs, there will still be density oscillations produced by the boundaries of an open ladder which only decay slowly (with a power law) into the ladder. Detailed predictions can be made about these using bosonization generalizing the approach of Ref. (8,9) for the spin chain and Hubbard chain to the case of the ladder. These oscillations provide a further check on the theory. Thus consider again the $4 k_{F}$ term in the density operator, of Eq. 2.10):

$$
n_{j}=A e^{-2 i \pi n j} e^{-2 i \sqrt{\pi} \theta_{+\rho}}+\text { h.c. }
$$

where $A$ is a constant into which we have adsorbed $<e^{-2 i \sqrt{\pi} \theta_{+\sigma}}>$. As we discussed in Sec. II, this $4 k_{F}$ term in the continuum representation of $n_{j}$, determines the leading behavior of the density-density correlation function at long distances since any $2 k_{F}$ terms decay exponentially. For an infinite system, the correlation function of the exponential operator appearing in Eq. (5.1), behaves as:

$$
<e^{2 i \sqrt{\pi} \theta_{+\rho}}(r) e^{-2 i \sqrt{\pi} \theta_{+\rho}}(0)>=c|r|^{-2 K_{+\rho}}
$$

where $c$ is a constant, with dimensions of (length) ${ }^{2 K_{+\rho}}$. Decomposing $\theta=\phi_{R}-\phi_{L}$, we may write this correlation function as a square of two identical factors, the correlation functions of $\exp \left[2 i \sqrt{\pi} \phi_{R / L}\right]$. It thus follows that the oscillating term in the density correlation function is:

$$
<n(r) n(0)>\rightarrow \cos (2 \pi n r+\alpha) 2 c|A|^{2}|r|^{-2 K_{+\rho}} .
$$

Now consider the semi-infinite system $(r>0)$ with one free boundary condition. As remarked in Sec. II, this corresponds to a b.c. on the boson field given in Eq. (2.23), or equivalently:

$$
\phi_{L}(t, 0)=\phi_{R}(t, 0)+\text { constant. }
$$

Now using the fact that $\phi_{R}$ is a function of $v t-r$ only and $\phi_{L}$ a function of $v t+r$ only, we see that this equation implies that we may regard $\phi_{R}$ as the analytic continuation of $\phi_{L}$ to the negative axis:

$$
\phi_{R}(r)=\phi_{L}(-r)+\text { constant. }
$$

Thus the expectation value of $n(r)$ reduces essentially to the square root of the correlation function $\langle n(r) n(0)\rangle$ :

$$
<n_{j}>\rightarrow 2 \sqrt{c}|A| \cos (2 \pi n j+\beta)(2 j)^{-K_{+\rho}} .
$$

The wave-vector of the Friedel oscillations is the same as the wave-vector governing the long-distance oscillations in the correlation function. No Friedel oscillations occur at wave-vector $2 k_{F i}$ as they would for the non-interacting system or more generally in phases where, for example, all 4 bosons are gapless. The exponent governing the decay of the Friedel oscillations is $K_{+\rho}, 1 / 2$ the exponent governing the decay of density correlations. Furthermore, the amplitude is simply the square root of the amplitude of the density correlation function (multiplied by $2^{1 / 2-K}$ ). Finally, we may readily generalize Eq. 5.6 to the case of a finite chain of length $L$, by a standard conformal transformation:

$$
<n_{j}>\rightarrow \frac{2 \sqrt{c}|A| \cos (2 \pi n j+\beta)}{[(2 L / \pi) \sin (\pi j / L)]^{K_{+\rho}}} .
$$




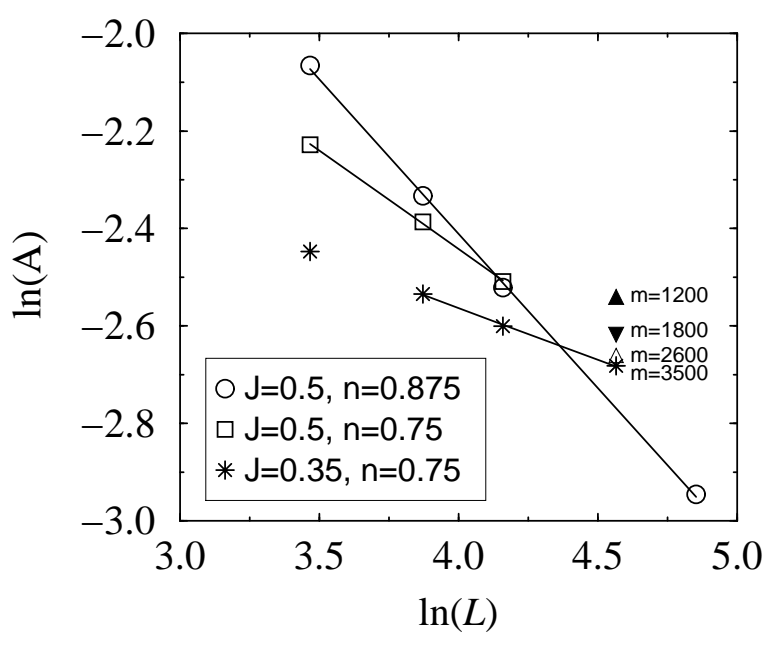

FIG. 11: The amplitude, $A$, of the Friedel oscillations in the center of the system, as a function of the length, $L$. The resulting slopes are $-K_{+\rho}$ and are given in Table III. The triangles show results for $L=64$ as a function of the number of block states kept, $m$, for $J=.35, n=.75$. One can see the very slow convergence of the amplitude with the number of states kept.

The decay of Friedel oscillations allows a way of determining the critical exponent $K_{+\rho}$ which is alternative to measuring directly the long distance behavior of the density correlations. Indeed this latter measurement becomes quite difficult with open b.c.'s due to the necessity of eliminating boundary effects.

We emphasize that the wave-vector of these Friedel oscillations $2\left(k_{F e}+k_{F o}\right)=2 \pi n$, or equivalently $2 \pi x$ where $x \equiv 1-n$ is the hole density, is a characteristic feature of the particular C1S0 phase that we are assuming. More correctly, this is the minimum Friedel oscillation wavevector since higher harmonics are also expected to occur. This minimum oscillation wave-vector would be different in a different phase. This wave-vector corresponds to 2 holes per wave-length and is the same wave-vector that would occur for a 1-component spinless hard core bose gas, which is an approximate description of the C1S0 phase in which hole pairs are assumed to form tightly bound rung singlets.6

An immediate consequence of Eq. (5.7) is that the amplitude of the density oscillations near the center of a finite chain scales as $L^{-K_{+\rho}}$. We show a log-log plot of this amplitude versus length in Fig. 11. Fitting to a straight line allows a determination of $K_{+\rho}$. The corresponding values of $K_{+\rho}$ determined in this way are shown in Table 11. We see that they are roughly comparable to the values obtained from the finite size spectrum.

In Figs. 12 and 13 we show the Friedel oscillations in the density at site $l$, for two different values of $(n, J)$, fitted to Eq. (5.7) with $K_{+\rho}$ taken as a free parameter. The agreement is fair although the presence of corrections due to irrelevant operator effects is evident. In both cases the value of $K_{+\rho}$ so determined is in rough agreement with the values in the third column of Table I, determined from the finite size spectrum.

We emphasize that these formulas are true very generally for Luttinger liquids with a single gapless charge boson. In particular, they apply to the spinless single chain model. In the non-interacting case we may readily find the exact formula for the Friedel oscillations. For $N$ electrons on $L$ sites:

$$
\begin{aligned}
& <n_{j}>=\frac{2}{L+1} \sum_{m=1}^{N} \sin ^{2} \frac{\pi m j}{L+1} \\
& =\frac{N+1 / 2}{L+1}-\frac{\sin [2 \pi j(N+1 / 2) /(L+1)]}{2(L+1) \sin [(\pi j /(L+1)]} .
\end{aligned}
$$

For large $N$ and $L$ this can be approximated:

$$
<n_{j}>\approx n-\frac{\sin 2 \pi n j}{2 L \sin (\pi j / L)} .
$$

This has the expected form of Eq. (5.7) with $K=1$ and $\sqrt{c}|A|=1 / 2 \pi$. On the other hand, the $2 k_{F}$ part of the density correlation function at long distances is:

$$
<n_{j} n_{0}>\rightarrow \frac{\cos 2 \pi n j}{2 \pi^{2}|j|^{2}}
$$

which has the form of Eq. (5.3) with the same value of $\sqrt{c}|A|=1 / 2 \pi$.

We see that, not surprisingly, when the charge density correlations drop off slowly, so do the Friedel oscillations. In particular, this makes it difficult to determine numerically whether or not a CDW occurs at $n=3 / 4$, for example, by measuring density oscillations. If $J / t$ is such

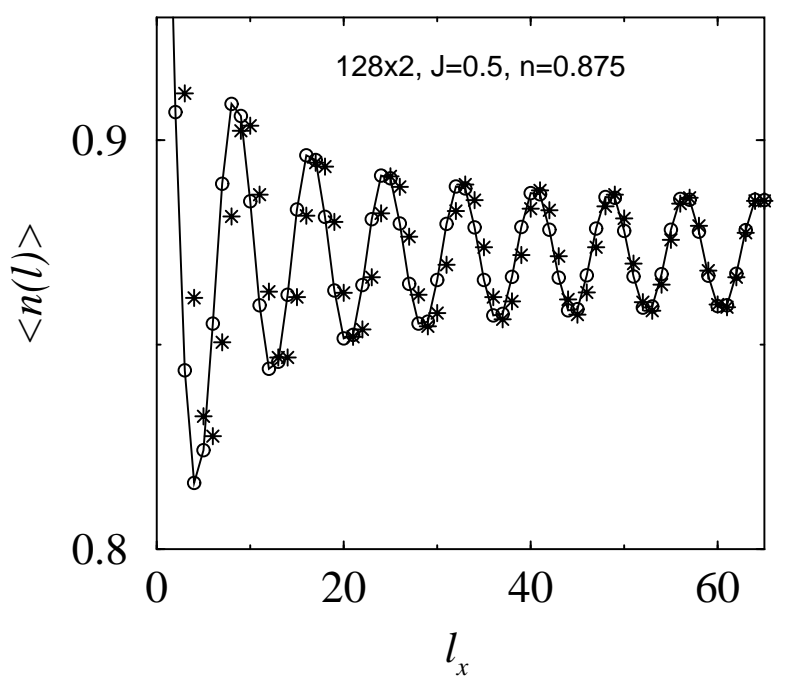

FIG. 12: Density at site $l$ from DMRG (* symbols) compared to Eq. (5.7) (circles and lines)for $(n, J)=(.5, .875)$ using $K_{+\rho}=.63$. 


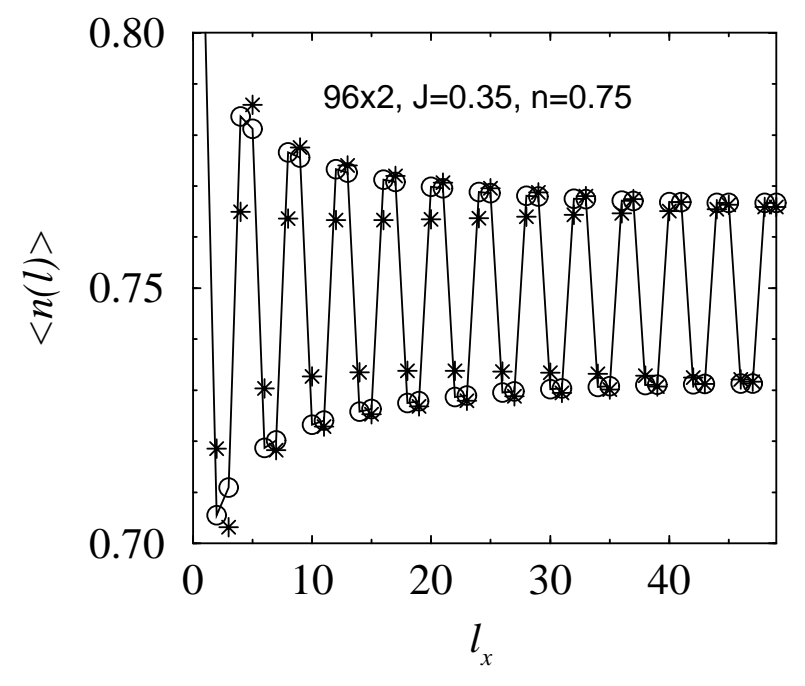

FIG. 13: Density at site $l$ from DMRG (* symbols) for $(n, J)=(.35, .75)$ compared to Eq. (5.7) (circles and lines) using $K_{+\rho}=0.33$.

that the system almost has a CDW then $K_{+\rho}$ will be only slightly greater than $1 / 8$. The extremely slow decay of the Friedel oscillations will be difficult to distinguish from a true CDW, where the oscillation amplitude goes to a non-zero constant far from the boundaries.

We now consider the single chain Hubbard and $t J$ models. The Hubbard model is known to be in a C1S1 phase for all densities (except $n=1$ ) and all $U>0$. Thus the low energy effective Hamiltonian contains both spin and charge bosons. (We use the same notation as for the 2leg ladder except that the \pm labels are no longer needed since there is only one type of charge boson and one type of spin boson.) The charge boson Hamiltonian is written exactly as in Eq. (2.6), in terms of the parameter $K_{\rho}$. The spin boson Hamiltonian also has exactly this form but with $K_{\sigma}=1$, as follows from $S U(2)$ spin rotation invariance. The pairing, $2 k_{F}$ and $4 k_{F}$ density operators now take the form:

$$
\begin{aligned}
\psi_{L \uparrow} \psi_{R \downarrow} & \propto e^{i \sqrt{2 \pi}\left(\phi_{\rho}-\theta_{\sigma}\right)} \\
e^{-2 i k_{F} x} \psi_{R \uparrow}^{\dagger} \psi_{L \uparrow} & \propto e^{-2 i k_{F} x} e^{-i \sqrt{2 \pi}\left(\theta_{\rho}+\theta_{\sigma}\right)} \\
e^{-4 i k_{F} x} \psi_{R \uparrow}^{\dagger} \psi_{L \uparrow} \psi_{R \downarrow}^{\dagger} \psi_{L \downarrow} & \propto e^{-4 i k_{F} x} e^{-2 i \sqrt{2 \pi} \theta_{\rho}} .
\end{aligned}
$$

Note that, in this case, we have formed a $4 k_{F}$ operator in which the spin boson doesn't appear. It then follows that the pair correlations, $2 k_{F}$ density correlations and $4 k_{F}$ density correlations decay with power law exponents:

$$
\begin{aligned}
\eta_{\text {pair }} & =1+1 / K_{\rho} \\
\eta_{2 k_{F}} & =1+K_{\rho} \\
\eta_{4 k_{F}} & =4 K_{\rho} .
\end{aligned}
$$

Open b.c.'s imply:

$$
\theta_{\rho}(0)=\text { constant }
$$

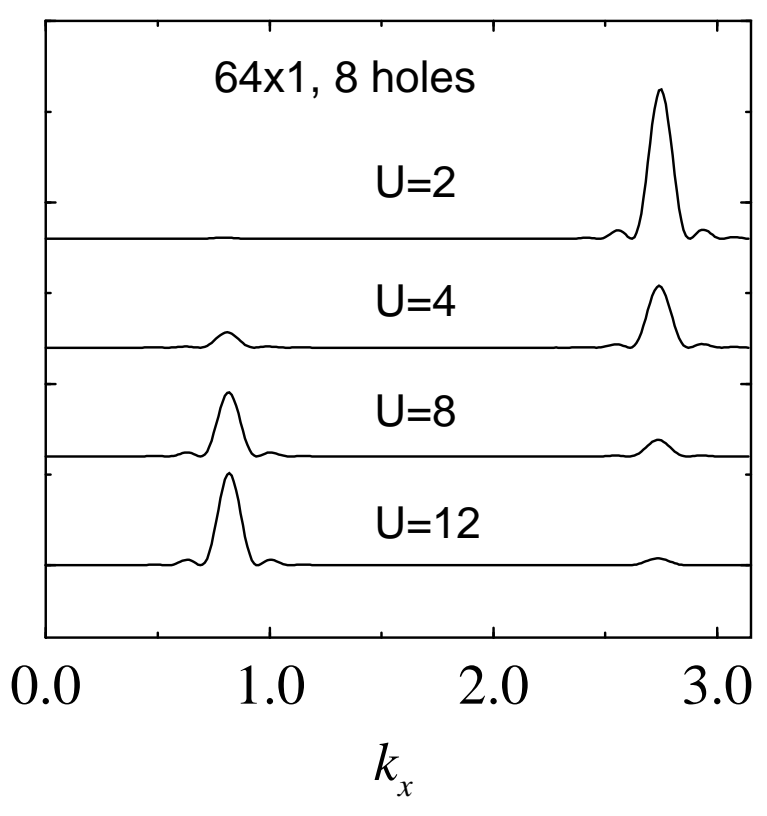

FIG. 14: Smoothed Fourier transform of the density, $n_{j}$ in the single chain Hubbard model at $7 / 8$ filling for various values of the repulsion strength, $U$.

$$
\theta_{\sigma}(0)=\text { constant. }
$$

It thus follows that the density will exhibit both $2 k_{F}$ and $4 k_{F}$ Friedel oscillations with different exponents:

$$
<n_{j}>\rightarrow n+\frac{A \cos (\pi n j+\alpha)}{j^{\left(1+K_{\rho}\right) / 2}}+\frac{B \cos (2 \pi n j+\beta)}{j^{2 K_{\rho}}},
$$

Where we have expressed the oscillation wave-vectors in terms of the electron density using the free electron result for electrons with spin on a single chain:

$$
2 k_{F}=\pi n
$$

The amplitudes, $A$ and $B$ are proportional to the square roots of the corresponding terms in the density correlation function and depend on density and $U$ (as does $K_{\rho}$ ).

It is interesting to consider the limit $U>>t$ where the no double occupancy constraint is present. If we consider some initial configuration of holons and spins, then the order of the spins along the chain can never change under nearest neighbor hopping processes consistent with no double occupancy. It follows that the charge dynamics must be identical to those of a system of non-interacting spinless fermions with the same density. That is to say, the charge dynamics is "spin blind". It follows that the density oscillations should not have the $\pi n$ term in this limit but only the $2 \pi n$ term as for spinless fermions [Eq. (5.9)]; i.e. that $A \rightarrow 0$ at $U \rightarrow \infty$. This argument also determines $K_{\rho}=1 / 2$ and $B=1 / 2 \pi$ in this limit. We remark that this argument is special to the single chain model with only nearest neighbor hopping. On a ladder, 
or even on a chain with next nearest neigbor hopping, spin rearrangement is possible without double occupancy by moving the electrons around or past each other.

In Fig. 14 we plot the Fourier transform of the density for the Hubbard model, at 7/8 filling, for various values of $U$. The same smoothing procedure was used that we mentioned in Sec. III. In this case $2 k_{F}=\pi n=7 \pi / 8$ and $4 k_{F}=7 \pi / 4$ or equivalently $\pi / 4$. Both $2 k_{F}$ and $4 k_{F}$ components are clearly visible and it is evident that the $2 k_{F}$ component vanishes at large $U$.

What does this imply about the (single chain) $t-J$ model? The $J \rightarrow 0$ limit of the $t-J$ model is identical to the $U \rightarrow \infty$ limit of the Hubbard model. Therefore, in that limit, the density oscillations have vanishing $2 k_{F}$ component. It is not completely obvious what happens at non-zero $J$. Insofar as this is the same as large but finite $U$, we would expect to have a small $2 k_{F}$ component to the density oscillations. Note however, that the
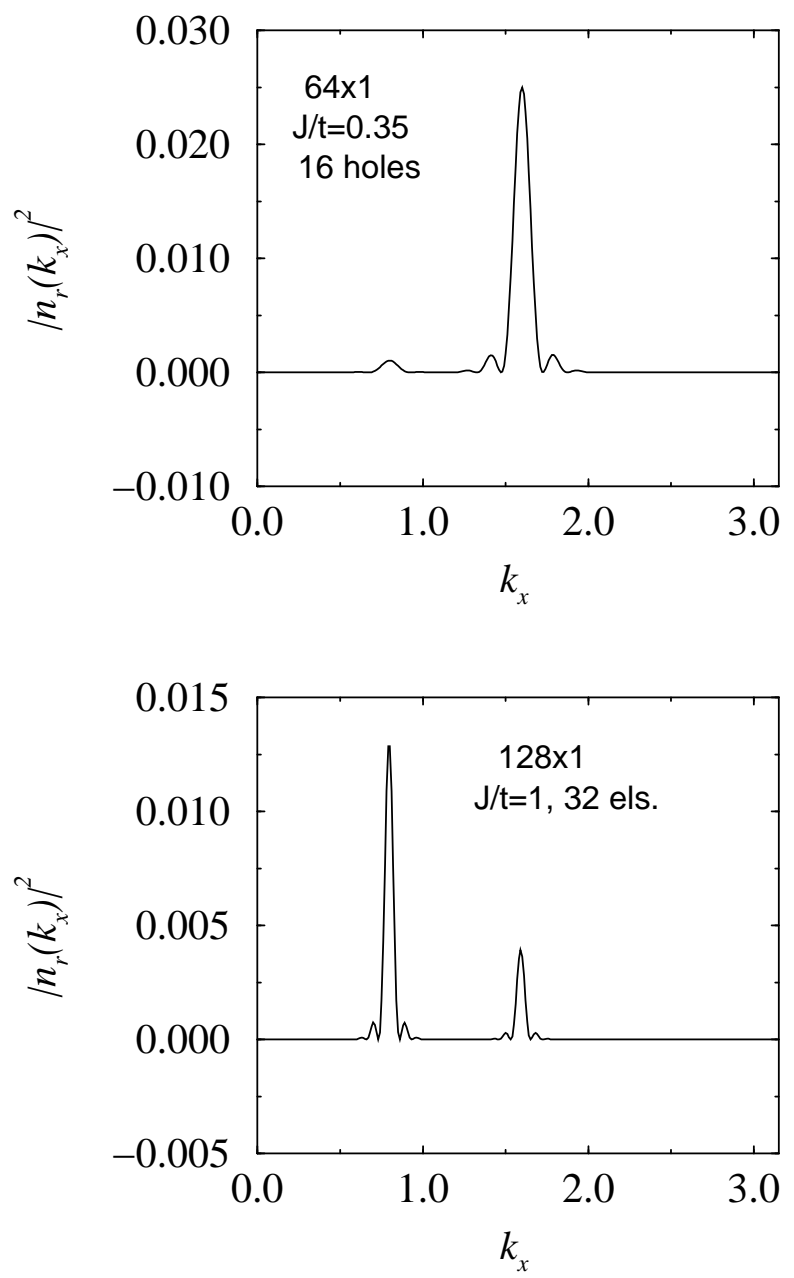

FIG. 15: Smoothed Fourier transform of the density, $n_{j}$ in the single chain $t J$ model at $1 / 4$ filling for a) $J / t=.35$ and b) $\mathrm{J}=1.0$, showing Friedel oscillations at $2 k_{F}=\pi / 4$ and $4 k_{F}=$ $\pi / 2$. no double occupancy constraint is enforced exactly in the $t-J$ model, by construction, whereas it is not in the finite $U$ Hubbard model. Thus we must understand whether it is the no double occupancy which is responsible for the vanishing $2 k_{F}$ oscillations or whether the vanishing of all spin rearrangement processes is neccessary. The argument in the previous paragraph seems to rely on the vanishing of spin rearrangement processes so we suspect that the Friedel oscillations should exhibit a $2 k_{F}$ component for any finite $J$. Of course, for small $J / t$ this is expected to be small. In Fig. 15 we show the Fourier transformed density for the single leg $t-J$ model at density $n=1 / 4$ for two values of $J / t$. Note that now $2 k_{F}=\pi / 4$ and $4 k_{F}=\pi / 2$. For smaller $J / t$ the $4 k_{F}$ oscillations clearly dominate although a small $2 k_{F}$ part is observed. At larger $\mathrm{J} / \mathrm{t}$, the oscillations at $2 k_{F}$ are larger than those at $4 k_{F}$.

Thus we expect $t J$ models to exhibit generic Friedel oscillations in all cases except for the special case of a single leg in the limit $J / t \rightarrow 0$ where the absence of spin rearrangement processes eliminates the lowest wavevector component of the oscillations.

The Friedel oscillation wave-vector can be a useful diagnostic of which phase a particular multi-leg $t J$ model is in. For instance, the minimum oscillation wave-vector, $2 k_{F e}+2 k_{F o}=2 \pi n$, is a characteristic of the particular C1S0 phase of the 2 leg ladder reviewed in Sec. II (in which the $\theta_{ \pm \sigma}$ and $\phi_{-\rho}$ fields are pinned). Other phases exhibit other oscillation wave-vectors. We expect such an analysis of DMRG results to be useful in determining the phase diagram of multi-leg ladders.

\section{Acknowledgments}

DJS would like to thank T. Giamarchi for helpful discussions. DJS and IA acknowlege the hospitality of the ITP, University of California, Santa Barbara where this work was initiated. SRW acknowledges support from the NSF under grant No. DMR98-70930; IA acknowleges support from the NSF Grant No. PHY99-07949 (ITP) and NSERC of Canada; DJS acknowledges support from the NSF under grant No. PHY99-07949 (ITP) and grant No. DRM98-17242 (DJS). 
* Electronic address: srwhite@.uci.edu

$\dagger$ Electronic address: affleck@physics.bu.edu; On leave from Canadian Institute for Advanced Research and Department of Physics and Astronomy, University of British Columbia, Vancouver, BC, Canada, V6T 1Z1

‡ Electronic address: djs@vulcan.physics.ucsb.edu

1 M. Fabrizio, Phys. Rev. B37, 325 (1993).

2 H.J. Schulz, Phys. Rev. B53, 2959 (1996).

${ }^{3}$ L. Balents and M.P.A. Fisher, Phys. Rev. B53, 12133 (1996).

4 E. Orignac and T. Giamarchi, Phys. Rev. B56, 7167 (1997).

5 R.M. Noack, S.R. White and D.J. Scalapino, Phys. Rev. Lett. 73, 882 (1994).

6 T. Siller, M. Troyer, T.M. Rice and S.R. White, Phys. Rev. B63, 195106 (2001).

7 C.A. Hayward and D. Poilblanc, Phys. Rev. B53, 11721 (1996).
8 S. Eggert and I. Affleck, Phys. Rev. Lett. 75, 934 (1995).

9 R. Egger and H. Grabert, Phys. Rev. Lett. 75, 3505 (1995).

10 S.R. White and D.J. Scalapino, Phys. Rev. B61, 6320 (2000).

11 H.J. Schulz, Phys. Rev. B59, 2471 (1999).

12 H.J. Schulz, Phys. Rev. B22, 5274 (1980).

13 T. Giamarchi, Physica B230, 975 (1997).

14 S. Eggert and I. Affleck, Phys. Rev. B46, 10866 (1992).

15 M Yamanaka, M. Oshikawa and I. Affleck, Phys. Rev. Lett. 79, 1110 (1997).

16 M. Vekic and S.R. White, Phys. Rev. Lett. 71, 4283 (1993); see Eq. (4) and (10).

17 D.J. Scalapino, S.R. White and I. Affleck, Phys. Rev. B64, 100506 (2001). J.B. Marston and A. Sudbo, condmat/0103120

18 S. Rommer, S.R. White and D.J. Scalapino, Phys. Rev. B61, 14701 (1997). 